\title{
ANÁLISE DE PARÂMETROS FÍSICO-QUÍMICOS DE EFLUENTE DE SUINOCULTURA MANEJADO COM PLANTAS AQUÁTICAS
}

Camila Dias Pinaffi; Carlos Henrique dos Santos

Universidade do Oeste Paulista, Mestrado em Meio Ambiente e Desenvolvimento Regional, Presidente Prudente, SP. E-mail: camila pinaffi@hotmail.com.

\section{RESUMO}

As plantas aquáticas representam uma possibilidade de manejo de efluentes de suinocultura. $\mathrm{O}$ objetivo do estudo foi analisar os parâmetros físico-químicos deste efluente manejado com as plantas aquáticas Eichhornia crassipes, Pistia stratiotes e Salvinia auriculata. O experimento ocorreu no Campus II/UNOESTE. Os tratamentos foram caracterizados como: T1 = Testemunha apenas 50\% DLS; T2 $=50 \%$ DLS + E. crassipes; T3 $=50 \%$ DLS + P. stratiotes $; \mathrm{T} 4=50 \% \mathrm{DLS}+S$. auriculata. $\mathrm{O}$ experimento foi estruturado em delineamento em blocos casualizados, com quatro repetições. Foram analisados: $\mathrm{pH}$, oxigênio dissolvido, condutividade elétrica e turbidez. Os resultados foram submetidos à análise de variância e ao Teste de Tukey, 5\% de probabilidade. Os valores de $\mathrm{pH}$ nos tratamentos com plantas apresentaram valores adequados, as concentrações de OD foram maiores no testemunha, houve redução da condutividade elétrica e a redução da turbidez foi maior nos tratamentos com plantas.

Palavras-chave: macrófitas, dejetos suínos, impacto ambiental.

\section{ANALYSIS OF PHYSICO-CHEMICAL PARAMETERS OF SUINOCULTURE EFFLUENT MANAGED WITH AQUATIC PLANTS}

\begin{abstract}
Aquatic plants represent a possibility of handling swine effluents. The objective of the study was to analyze the physico-chemical parameters of this effluent handled with the aquatic plants Eichhornia crassipes, Pistia stratiotes and Salvinia auriculata. The experiment took place in Campus II / UNOESTE. The treatments were characterized as: T1 = Witness - only $50 \%$ DLS; T2 = $50 \% \mathrm{DLS}+$ E. crassipes; $\mathrm{T3}=50 \% \mathrm{DLS}+P$. stratiotes $; 4=50 \% \mathrm{DLS}+S$. auriculata . The experiment was structured in a randomized block design, with four replications. The following parameters were analyzed: $\mathrm{pH}$, dissolved oxygen, electrical conductivity and turbidity. The results were submitted to analysis of variance and Tukey's test, $5 \%$ probability. The values of $\mathrm{pH}$ in the treatments with plants presented adequate values, the concentrations of OD were higher in the control, there was a reduction of electrical conductivity and the reduction of turbidity was higher in the treatments with plants.
\end{abstract}

Keywords: macrophytes, swine manure, environmental impact. 


\section{INTRODUÇÃO}

Com a gradativa ascensão da população mundial, a produção de alimentos torna-se uma necessidade eminente. Nessa perspectiva, a suinocultura vem atingindo patamares relevantes, pois representa um setor de grande importância econômica e social (GONZATTO et al., 2013). Com este avanço, o volume de dejetos (efluentes) aumentou, causando grandes problemas ambientais, principalmente pela disposição direta desses dejetos no solo. Conforme Zhang et al. (2012), esta prática executada sem o gerenciamento correto pode resultar na contaminação dos compartimentos do meio ambiente, pois as áreas agrícolas para a eliminação são limitadas.

Diante disto, verifica-se a necessidade de estudos em busca de um manejo adequado dos efluentes de suinocultura e de sistemas de tratamento que assegurem e promovam benefícios na redução do seu poder poluente. Uma alternativa que tem se revelado eficiente e de baixo custo é a fitorremediação, técnica capaz de empregar sistemas vegetais fotossintetizantes e sua microbiota, com o fim de desintoxicar ambientes poluídos (DINARDI et al., 2003).

As macrófitas aquáticas, por necessitarem de altas concentrações de nutrientes para seu desenvolvimento, destacam-se nesta modalidade de tratamento, sendo capazes de melhorar a qualidade das águas poluídas por meio dos mecanismos de absorção e assimilação. As plantas são capazes de remover nutrientes, muitos dos quais ficam presos no sistema por sucessivos ciclos de crescimento, morte e decomposição (KADLEC et al., 2005), além de formarem uma densa rede capaz de reter as mais finas partículas em suspensão (LEITÃO JÚNIOR et al., 2007).

Neste sentido, o presente estudo tem como objetivo analisar os parâmetros físico-químicos $(\mathrm{pH}$, oxigênio dissolvido, condutividade elétrica e turbidez) de efluente de suinocultura manejado com as plantas aquáticas Eichhornia crassipes, Pistia stratiotes e Salvinia auriculata.

\section{MATERIAL E MÉTODOS}

O experimento foi desenvolvido em área localizada a $475 \mathrm{~m}$ de altitude, com clima definido como Aw, conforme classificação de Köppen, no Campus II da Universidade do Oeste Paulista (UNOESTE), Presidente Prudente - SP.

Para a composição dos tratamentos foi utilizado efluentes da suinocultura, caracterizados físico-quimicamente in natura (Tabela 1), do centro zootécnico, Campus II/UNOESTE. Os tratamentos foram constituídos de 16 recipientes PVC $\left(0,32 \mathrm{~m}^{3}\right)$, e caracterizados como: $\mathrm{T} 1=$ Testemunha - $50 \%$ de DLS e $50 \%$ de água; T2 = 50\% de DLS e 50\% de água + E. crassipes; T3 = 50\% de DLS e $50 \%$ de água $+P$. stratiotes; $\mathrm{T4}=50 \%$ de DLS e $50 \%$ de água $+S$. auriculata, distribuídos em delineamento em blocos casualizados, em parcelas subdivididas, com quatro repetições. As parcelas constituem os tratamentos (sem e com a presença de plantas aquáticas) e as subparcelas, os períodos de coleta do efluente para análise química $(0,7,14,21$ e 30 dias).

Tabela 1. Valores médios das amostras de efluente in natura destinadas à caraterização físicoquímica

\begin{tabular}{lc}
\hline \multicolumn{1}{c}{ Parâmetros } & Valores \\
\hline $\mathrm{pH}$ & $7,39 \pm 0,12$ \\
$\mathrm{OD}\left(\mathrm{mg} \mathrm{L}^{-1}\right)$ & $0,65 \pm 0,03$ \\
Condutividade elétrica $\left(\mu \mathrm{S} \mathrm{cm}^{-1}\right)$ & $4480,06 \pm 0,05$ \\
Turbidez (NTU) & $642,17 \pm 96,84$ \\
\hline
\end{tabular}

As plantas aquáticas foram coletadas em propriedades pertencentes à região do Oeste Paulista, São Paulo. No local do experimento, as plantas foram submetidas à aclimatação ambiental em caixas com água, durante uma semana. Posterior a este período, iniciou-se o 
tratamento, e a quantidade de plantas inseridas em cada caixa foi determinada para manter uma ocupação de $80 \%$ das unidades experimentais, conforme relatos de Henry-Silva e Camargo (2008).

Durante o período experimental (março e abril de 2016), foram coletadas semanalmente 16 amostras do efluente, sendo uma amostra por caixa, acondicionada em garrafas plásticas descartáveis com tampa lacre de $500 \mathrm{ml}$.

As análises realizadas foram: potencial hidrogeniônico $(\mathrm{pH})$, oxigênio dissolvido (OD), condutividade elétrica (CE) e turbidez, sendo utilizados pHmetro digital de bancada, marca Micronal, oxímetro portátil, marca Hanna Instruments, condutivímetro digital de bancada, marca Hanna Instruments, turbidímetro portátil microprocessado, marca Quimis, respectivamente.

A eficiência dos tratamentos na redução da condutividade elétrica e turbidez foi calculada conforme a Equação 1:

$$
E(\%)=\frac{C i-C f}{C i} \times 100
$$

Em que: $\mathrm{E}=$ Eficiência de remoção (\%); $C i=$ Concentração inicial $\left(\mathrm{mg} \mathrm{L}^{-1}\right) ; C f=$ Concentração final $\left(\mathrm{mg} \mathrm{L}^{-1}\right)$.

Considerou-se os valores de concentração inicial, a média obtida nas análises da primeira coleta (0 dia), e os de concentração final, a média obtida nas análises da última coleta (30 dias).

Os resultados foram submetidos à análise de variância e a comparação das médias pelo Teste de Tukey, ao nível de 5\% de probabilidade.

\section{RESULTADOS E DISCUSSÃO}

Constatou-se um incremento dos valores de $\mathrm{pH}$ (Tabela 2) nos tratamentos ao longo dos 30 dias de experimentação, o que também pode ser explicado pela elevada perda de água pela evapotranspiração e consequentemente a concentração de bases no efluente.

Tabela 2. Médias de interação entre tratamentos e período de coleta, para pH dos tratamentos com efluente de suinocultura, na presença de E. crassipes, $P$. stratiotes, S. auriculata

Potencial Hidrogeniônico (pH)

\begin{tabular}{lccccc} 
Tratamentos & \multicolumn{5}{c}{ Período de Coleta } \\
Início & $\mathbf{7}$ dias & $\mathbf{1 4}$ dias & $\mathbf{2 1}$ dias & $\mathbf{3 0}$ dias \\
\cline { 2 - 6 } Testemunha & $7,36 \mathrm{aC}$ & $7,86 \mathrm{aC}$ & $8,03 \mathrm{aB}$ & $9,14 \mathrm{aA}$ & $9,31 \mathrm{aA}$ \\
E. crassipes & $7,35 \mathrm{aA}$ & $7,54 \mathrm{bcA}$ & $7,50 \mathrm{bA}$ & $7,28 \mathrm{cA}$ & $7,51 \mathrm{cA}$ \\
P. stratiotes & $7,32 \mathrm{aC}$ & $7,71 \mathrm{abA}$ & $7,62 \mathrm{bAB}$ & $7,37 \mathrm{cBC}$ & $7,63 \mathrm{bcAB}$ \\
S. auriculata & $7,34 \mathrm{aD}$ & $7,42 \mathrm{cCD}$ & $7,66 \mathrm{bBC}$ & $8,16 \mathrm{bA}$ & $7,80 \mathrm{bB}$ \\
\hline
\end{tabular}

dms para colunas $=0,26 ; \mathrm{CV}(\%)=1,79$ classificação com letras minúsculas. dms para linhas $=0,28 ; \mathrm{CV}(\%)=1,78$ classificação com letras maiúsculas. Foi aplicado o Teste Tukey ao nível de $5 \%$ de probabilidade

Observa-se ainda na Tabela 2, que os valores de $\mathrm{pH}$ obtidos nos tratamentos com as macrófitas aquáticas são inferiores aos obtidos na testemunha, sendo que aqueles observados no

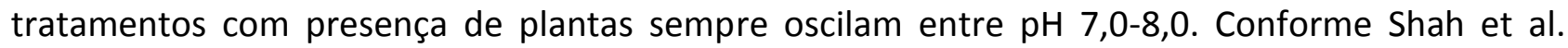
(2015), um pH de 6,0-9,0 é o mais adequado para o desempenho de macrófitas aquáticas. De acordo com a Resolução CONAMA 430/2011, os valores de pH do efluente de qualquer fonte poluidora, para serem lançados diretamente no corpo receptor, devem estar entre 5,0 e 9,0. Desta 
forma, os resultados encontrados nos tratamentos com as espécies vegetais estão de acordo com os exigidos pela legislação, embora o tratamento testemunha (sem plantas) tenha ultrapassado esse limite a partir do período de 21 dias.

Na Tabela 3 observa-se acréscimo da concentração de OD dissolvido ao longo dos 30 dias, sendo que este aumento foi maior no período de 14 e 21 dias, onde a cobertura vegetal não havia atingido toda a superfície do reservatório. Semelhantemente ao observado no primeiro ciclo, os valores do tratamento testemunha foram maiores que os demais tratamentos com espécies vegetais. Reddy e DeBusk (1985) também verificaram que, em sistemas de tratamento de efluentes com macrófitas aquáticas de grandes lâminas foliares, como $E$. crassipes, $P$. stratiotes e H. umbellata, em decorrência do sombreamento e da decomposição da matéria orgânica por organismos aeróbios, as concentrações de OD foram baixas (2,4-3,9 $\left.\mathrm{mg} \mathrm{L}^{-1}\right)$.

É interessante destacar que as concentrações de OD registradas no tratamento com $S$. auriculata foram maiores quando comparadas às demais espécies (Tabela 3). Provavelmente, por possuir pequenas lâminas foliares e talvez, por restringir menos a entrada de luz no efluente, permitiu melhor oxigenação do efluente.

Tabela 3. Médias de interação entre tratamentos e período de coleta, para OD dos tratamentos com efluente de suinocultura, na presença de E. crassipes, P. stratiotes, S. auriculata

\begin{tabular}{cccccc}
\hline \multicolumn{5}{c}{ Oxigênio Dissolvido $\left(\mathbf{m g ~ L}^{-\mathbf{1}}\right.$ ) } \\
\hline \multirow{2}{*}{ Tratamentos } & $\mathbf{0 ~ d i a}$ & $\mathbf{7}$ dias & 14 dias & 21 dias & 30 dias \\
\cline { 2 - 6 } Testemunha & $0,55 \mathrm{aB}$ & $2,02 \mathrm{aB}$ & $7,31 \mathrm{aA}$ & $7,68 \mathrm{aA}$ & $6,96 \mathrm{aA}$ \\
E. crassipes & $0,62 \mathrm{aB}$ & $1,06 \mathrm{aB}$ & $4,05 \mathrm{bA}$ & $5,68 \mathrm{abA}$ & $2,85 \mathrm{bAB}$ \\
P. stratiotes & $0,83 \mathrm{aB}$ & $0,96 \mathrm{aB}$ & $6,78 \mathrm{aA}$ & $3,69 \mathrm{bB}$ & $2,37 \mathrm{bB}$ \\
S. auriculata & $0,77 \mathrm{aB}$ & $1,05 \mathrm{aB}$ & $6,26 \mathrm{abA}$ & $6,24 \mathrm{abA}$ & $4,59 \mathrm{abA}$ \\
\hline
\end{tabular}

$\mathrm{dms}$ para colunas $=2,57 ; \mathrm{CV}(\%)=40,04$ classificação com letras minúsculas. dms para linhas $=2,90 ; \mathrm{CV}(\%)=28,43$ classificação com letras maiúsculas. Foi aplicado o Teste Tukey ao nível de $5 \%$ de probabilidade

Nota-se pequena redução da condutividade elétrica no decorrer do tempo de avaliação (Tabela 4). Observa-se, ainda, que não houve diferença estatística entre a $E$. crassipes e $P$. stratiotes ao final dos 30 dias, e que a redução da CE foi mais expressiva no tratamento testemunha, que não apresentava espécies vegetais.

Tabela 4. Médias de interação entre tratamentos e período de coleta, para CE dos tratamentos com efluente de suinocultura, na presença de E. crassipes, $P$. stratiotes, S. auriculata

\begin{tabular}{lcccccc}
\hline \multicolumn{7}{c}{ Condutividade Elétrica $\left(\boldsymbol{\mu S} \mathbf{~ c m}^{-\mathbf{1}}\right)$} \\
\hline \multirow{2}{*}{ Tratamentos } & $\mathbf{0 ~ d i a}$ & $\mathbf{7}$ dias & 14 dias & 21 dias & $\mathbf{3 0}$ dias & E (\%) \\
\cline { 2 - 7 } Testemunha & $1319,50 \mathrm{aA}$ & $1160,00 \mathrm{bB}$ & $979,25 \mathrm{bC}$ & $645,25 \mathrm{cD}$ & $767,00 \mathrm{bD}$ & 41,9 \\
E. crassipes & $1358,00 \mathrm{aA}$ & $1321,75 \mathrm{abA}$ & $1288,50 \mathrm{aA}$ & $858,00 \mathrm{abB}$ & $969,75 \mathrm{aB}$ & $\mathbf{2 8 , 6}$ \\
P. stratiotes & $1293,25 \mathrm{aA}$ & $1270,50 \mathrm{abA}$ & $1205,25 \mathrm{aAB}$ & $795,50 \mathrm{bcC}$ & $1062,50 \mathrm{aB}$ & 17,8 \\
S. auriculata & $1356,50 \mathrm{aA}$ & $1422,25 \mathrm{aA}$ & $1327,50 \mathrm{aA}$ & $1005,00 \mathrm{aB}$ & $937,25 \mathrm{abB}$ & $\mathbf{3 0 , 9}$ \\
\hline
\end{tabular}

dms para colunas $=196,81 ; C V(\%)=6,50$ classificação com letras minúsculas. dms para linhas $=145,56 ; C V(\%)=15,47$ classificação com letras maiúsculas. Foi aplicado o Teste Tukey ao nível de $5 \%$ de probabilidade. E (\%): Eficiência de remoção

Possivelmente, neste período, a taxa de perda de água pela evapotranspiração foi maior, e de acordo com Sposito (2013) a absorção de água pelas plantas aquáticas, e também a perda pela 
evaporação direta para a superfície, proporciona concentração de bases no efluente e, consequentemente pouca expressividade na diminuição da condutividade elétrica. Ainda, no período de 30 dias foi constatada elevação da CE quando comparado ao de 21 dias para o tratamento com a espécie $P$. stratiotes (Tabela 4). Provavelmente as espécies vegetais já estavam em processo de decomposição, e segundo Martins e Pitelli (2005), o aumento da condutividade elétrica do efluente pode decorrer da liberação de nutrientes durante o processo de decomposição das plantas.

Em relação à turbidez, na Tabela 5 observa-se que a espécie $E$. crassipes apresentou melhores resultados no período de 21 dias, com uma eficiência de remoção de 78,1\%. A $P$. stratiotes demonstrou resultados mais eficazes na redução da turbidez no período de 7 a 21 dias, sem diferença estatística entre eles, sendo que a taxa de remoção em 7 dias foi de $80,4 \%$. Já a $S$. auriculata obteve menores valores de turbidez no período de 30 dias, alcançando uma eficiência de $75,4 \%$. Provavelmente, a eficiência dos sistemas com $E$. crassipes e $P$. stratiotes na redução da turbidez foi mais elevada pois, conforme Henry-Silva e Camargo (2008), o grande desenvolvimento radicular dessas duas espécies e à pouca profundidade dos tanques criaram condições favoráveis para a adsorção e precipitação do material particulado em suspensão. Ainda de acordo com Henry-Silva e Camargo (2008), a eficiência dos sistemas com E. crassipes e $P$. stratiotes na redução da turbidez do efluente de carcinicultura foi de $80,2 \%$ e $75,2 \%$, respectivamente.

Tabela 5. Médias de interação entre tratamentos e período de coleta, para turbidez dos tratamentos com efluente de suinocultura, na presença de E. crassipes, $P$. stratiotes, S. auriculata

\begin{tabular}{lcccccc}
\hline \multicolumn{7}{c}{ Turbidez (NTU) } \\
\hline \multirow{2}{*}{ Tratamentos } & $\mathbf{0 ~ d i a}$ & $\mathbf{7}$ dias & 14 dias & 21 dias & 30 dias & E (\%) \\
\cline { 2 - 7 } Testemunha & $243,00 \mathrm{aA}$ & $69,90 \mathrm{aC}$ & $103,82 \mathrm{aC}$ & $182,00 \mathrm{aB}$ & $183,00 \mathrm{aB}$ & $\mathbf{2 4 , 7}$ \\
E. crassipes & $235,75 \mathrm{aA}$ & $78,08 \mathrm{aBC}$ & $92,48 \mathrm{aBC}$ & $51,53 \mathrm{cC}$ & $131,75 \mathrm{abB}$ & 44,1 \\
P. stratiotes & $215,25 \mathrm{aA}$ & $42,28 \mathrm{aC}$ & $54,38 \mathrm{aBC}$ & $62,00 \mathrm{cBC}$ & $99,48 \mathrm{bcB}$ & 53,8 \\
S. auriculata & $252,33 \mathrm{aA}$ & $86,45 \mathrm{aBC}$ & $94,53 \mathrm{aBC}$ & $126,05 \mathrm{bB}$ & $62,10 \mathrm{cC}$ & 75,4 \\
\hline
\end{tabular}

dms para colunas $=52,36 ; \mathrm{CV}(\%)=22,01$ classificação com letras minúsculas. dms para linhas $=54,41 ; \mathrm{CV}(\%)=25,01$ classificação com letras maiúsculas. Foi aplicado o Teste Tukey ao nível de $5 \%$ de probabilidade. E (\%): Eficiência de remoção

\section{CONCLUSÕES}

A redução nos valores dos parâmetros físico-químicos do efluente de suinocultura pelas plantas aquáticas ocorreu de modo distinto para cada espécie e entre os períodos de coleta semanal, sendo que:

- Os valores de $\mathrm{pH}$ nos tratamentos com plantas aquáticas apresentaram valores adequados à legislação.

- As concentrações de OD foram superiores no tratamento testemunha.

- Houve redução da condutividade elétrica conforme os períodos de coleta.

- A redução da turbidez foi maior nos tratamentos com plantas aquáticas.

\section{REFERÊNCIAS}

CONAMA - CONSELHO NACIONAL DO MEIO AMBIENTE. Resolução Conama no 430 de 13 de maio de 2011. Dispõe sobre as condições e padrões de lançamento de efluentes, complementa e altera 
a Resolução n. 357, de 17 de março de 2005, do Conselho Nacional do Meio Ambiente-CONAMA. Diário Oficial da União, Brasília, DF, n. 92, de 16/05/2011, p.89.

DINARDI, A. L. et al. Fitorremediação. In: FÓRUM DE ESTUDOS CONTÁBEIS, 3., 2003, Rio Claro. Anais... [S.I.]: [s.n.], 2003.

GONZATTO, R. et al. Volatilização de amônia e emissão de óxido nitroso após aplicação de dejetos líquidos de suínos em solo cultivado com milho. Ciência Rural, Santa Maria, v. 43, n. 9, p. 15901596, 2013. https://doi.org/10.1590/S0103-84782013000900009

HENRY-SILVA, G. G.; CAMARGO, A. F. M. Tratamento de efluentes de carcinicultura por macrófitas aquáticas flutuantes. Revista Brasileira de Zootecnia, v. 37, n. 2, p. 181-188, 2008. https://doi.org/10.1590/S1516-35982008000200002

KADLEC, R. H. et al. Nitrogen spiraling in subsurface-flow constructed wetlands: implications for treatment response. Ecological Engineering, v. 25, p. 365-381, 2005. https://doi.org/10.1016/i.ecoleng.2005.06.009

LEITÃO JÚNIOR, A. M. et al. Sistema de tratamento alternativo de efluentes utilizando macrófitas aquáticas: um estudo de caso do tratamento de efluentes frigoríficos por Pistia stratiotes e Eichhornia crassipes. Caminhos de Geografia, Uberlândia, v. 8, n. 23, ed. esp., p. 8-19, 2007.

MARTINS, A. T; PITELLI, R. A. Efeitos do manejo de Eichhornia crassipes sobre a qualidade da água em condições de mesocosmos. Planta Daninha, Viçosa, v. 23, n. 2, p. 233-242, 2005.

REDDY, K. R.; DEBUSK, T. A. Nutrient removal potential of selected aquatic macrophytes. Journal of Environmental Quality, v. 14, n. 4, p. 459-462, 1985. https://doi.org/10.2134/jeq1985.00472425001400040001x

SHAH, M. et al. Performance assessment of aquatic macrophytes for treatment of municipal wastewater. Journal of the South African Institution of Civil Engineering, v. 57, n. 3, p. 18-25, 2015. https://doi.org/10.17159/2309-8775/2015/V57N3A3

SPOSITO, T. H. N. Parâmetros físico-químicos do efluente de ETE do distrito urbano de Montalvão/SP manejado com aguapé. 2013. 92 f. Dissertação (Mestrado em Agronomia) Universidade do Oeste Paulista, Presidente Prudente.

ZHANG, Y. G. et al. Pig production in subtropical agriculture. Journal of the Science of Food and Agriculture, v. 92, n. 5, p. 1016-1024, 2012. https://doi.org/10.1002/isfa.4679 